\title{
Real-World Comparative Effectiveness and Cost Comparison of Thromboprophylactic Use of Enoxaparin versus Unfractionated Heparin in 376,858 Medically III Hospitalized US Patients
}

\author{
S. Phani Veeranki ${ }^{1,2,7} \cdot$ Zhimin Xiao $^{3,8} \cdot$ Andrée Levorsen $^{4}$ (D) $\cdot$ Meenal Sinha ${ }^{1} \cdot$ Bimal R. Shah $^{5,6}$
}

Accepted: 13 November 2020 / Published online: 14 December 2020

(c) The Author(s) 2020

\begin{abstract}
Background Venous thromboembolism (VTE) is a serious complication in medically ill inpatients. Enoxaparin or unfractionated heparin (UFH) thromboprophylaxis has been shown to reduce VTE in clinical trials; however, comparative effectiveness and differences in hospital costs are unknown in US hospital practice.

Objective This study compared clinical and economic outcomes between enoxaparin and UFH thromboprophylaxis in medically ill inpatients.

Methods A retrospective cohort study was conducted using the Premier Healthcare Database between 1 January 2010 and 30 September 2016. Inpatients aged $\geq 18$ years with a $\geq 6$-day hospital stay for serious medical conditions were included. Two patient groups receiving thromboprophylaxis were identified during hospitalization: one receiving enoxaparin and other receiving UFH. Regression models were constructed to compare VTE events, in-hospital mortality, pulmonary embolism (PE)-related mortality, major bleeding, and total hospital costs during both the index hospitalization and the 90-day readmission period between the two groups.

Results A total of 242,474 and 134,384 inpatients received enoxaparin or UFH for thromboprophylaxis, respectively. Compared with UFH prophylaxis, enoxaparin was significantly associated with 15\%, 9\%, 33\%, and $41 \%$ reduced odds of VTE, in-hospital mortality, PE-related mortality, and major bleeding, respectively, during index hospitalization, and 10\% and 19\% reduced odds of VTE and bleeding, respectively, during the readmission period. Mean total hospital costs were significantly lower in patients receiving enoxaparin prophylaxis than in those given UFH.

Conclusions Thromboprophylaxis with enoxaparin was associated with significantly reduced in-hospital VTE events, death, and major bleeding and lower hospital costs compared with UFH in hospitalized medically ill patients.
\end{abstract}

\section{Key Points}

Thromboprophylaxis is recommended for medical inpatients at risk of venous thromboembolism.

Enoxaparin versus unfractionated heparin prophylaxis was associated with reduced in-hospital venous thromboembolism, death, and bleeding.

Prophylaxis with enoxaparin compared with unfractionated heparin was associated with lower hospital costs.

This work was presented in part at the XXVII Congress of the International Society on Thrombosis and Haemostasis, Melbourne, Australia, 6-10 July 2019.

Andrée Levorsen

andree.levorsen@sanofi.com

Extended author information available on the last page of the article

\section{Introduction}

Venous thromboembolism (VTE), manifesting as deep vein thrombosis (DVT) and/or pulmonary embolism $(\mathrm{PE})$, is a serious medical condition causing significant morbidity and mortality. It has been estimated that approximately 2014 US $\$ 12,000-15,000$ are required to treat one incident acute VTE event, and approximately 2014US $\$ 18,000-23,000$ to treat subsequent complications, resulting in an overall economic burden of 2014US\$7-10 billion annually in the USA [1]. Hospitalization for a serious medical condition is a transiently acquired risk factor associated with increased risk of VTE [2]. Medically ill inpatients, including those hospitalized for pulmonary diseases, circulatory disorders, infections, or cancer, often have prolonged hospital stays with periods of immobility and are found to have an at least tenfold increased risk of VTE [3-5]. An estimated 10-20\% of these patients 
develop VTEs in the absence of thromboprophylaxis [6, 7]. Thus, the American College of Chest Physicians and American Society of Hematology have recommended pharmacologic thromboprophylaxis for all hospitalized patients at risk of VTE $[8,9]$.

Low-molecular-weight heparin (LMWH) and unfractionated heparin (UFH) are the most commonly used anticoagulants for thromboprophylaxis in medically ill hospitalized patients. Meta-analyses have demonstrated that thromboprophylaxis with LMWH or UFH reduced the incidence of DVT by up to $67 \%$ and of PE by up to $74 \%$ [10-12]. In addition, clinical trials have reported that thromboprophylaxis with enoxaparin, the most commonly prescribed LMWH, was cost effective and significantly reduced VTE events in medically ill inpatients without increasing adverse events compared with UFH [13-17]. However, differences in clinical outcomes and hospital costs between them is largely unknown in current hospital practice in the USA. This study assessed the use of enoxaparin and UFH among hospitalized medically ill patients. Specifically, it sought to compare clinical and economic outcomes between medically ill patients who received either enoxaparin or UFH for VTE prophylaxis during their hospitalization.

\section{Methods}

\subsection{Study Design and Data Source}

A retrospective cohort study was conducted using the Premier Healthcare Database (PHD). The PHD is a large hospital-based, service-level, all-payer database representing approximately $25 \%$ of all annual admissions from diverse non-profit, non-governmental, community and teaching hospitals, and health systems from rural and urban areas in the USA [18]. At the time of this study, more than 850 hospitals contributed data to the PHD. Through a unique masked identifier, patients in the PHD can be tracked across inpatient and outpatient encounters to the same hospital system. Data extracted from standard hospital discharge files include patient demographics and disease status, admission and discharge diagnoses, information on date-stamped billed items at the departmental level for medications, services, procedures, laboratory tests, and diagnostic and therapeutic services, microbiology tests, and patient disposition and discharge health status. Information on medication utilization is available by day of patient hospital stay and includes dose, quantity, strength, and cost per dose. Data used for this study were de-identified, Health Insurance Portability and Accountability Act compliant, and considered exempt from institutional review board oversight as per 45 CFR 46.101(b)(4).

\subsection{Study Population}

Adult patients aged $\geq 18$ years with an inpatient admission between 1 January 2010 and 30 September 2016, a hospital stay of at least 6 days, and a primary or secondary admission or discharge International Classification of Diseases, Ninth Revision (ICD)-9 and Tenth Revision, Clinical Modification (-10-CM) code for circulatory disorders (ICD-9-CM: 390.xx-459.xx; ICD-10-CM: I00.xxx-I99.xxx), respiratory conditions (ICD-9-CM: 460.xx-519.xx; ICD-10-CM: J00. xxx-J99.xxx), infectious diseases (ICD-9-CM: 001.xx-139. $\mathrm{xx}$, 680.xx-686.xx, 730.xx, 996.xx-999.xx; ICD-10-CM: A00.xxx-B99.xxx, J02.xxx-J03.xxx, K11.xxx-K65.xxx, L00.xxx-L99.xxx, M46.xxx, M86.xxx-M90.xxx, T80. xxx-T88.xxx), or neoplasms (ICD-9-CM: 140.xx-239.xx; ICD-10-CM: C00.xxx-D49.xxx) were identified (index hospitalization period). To minimize the effect of bias or confounding, patients were excluded if they met certain criteria during the index hospitalization and 90 days preceding the index hospitalization admission (pre-index hospitalization period). The primary exclusion criteria included patients with VTE diagnoses and/or therapeutic use of anticoagulants during the pre-index hospitalization period and within the first 2 days of index hospitalization admission. Patients with receipt of mechanical methods of VTE prophylaxis, receipt of vitamin $\mathrm{K}$ antagonists, diagnoses with thrombophilic conditions or hemorrhagic disorders, active peptic ulcer, or any surgery during the pre-index and index hospitalization periods were excluded. Patients included in the final assessment received VTE prophylaxis with either enoxaparin or UFH. Patients who received any combination of enoxaparin and UFH or received another anticoagulant (including fondaparinux, dalteparin, and rivaroxaban) along with enoxaparin or UFH were excluded. Patients were followed for up to 90 days after index hospitalization discharge to capture study outcomes among those who were readmitted to the same hospital (readmission period).

\subsection{Study Measures}

The primary clinical outcome was the occurrence of VTE during index hospitalization and readmission periods. A VTE event was defined using either primary or secondary admission or discharge ICD-9/10-CM diagnosis codes for DVT (ICD-9-CM: 451.xx-453.xx; ICD-10-CM: I80. xxx-I82.xxx) and/or PE (ICD-9-CM: 415.1x; ICD-10-CM: I26.9x and T80.xxxx-T82.xxxx). Secondary clinical outcomes included in-hospital mortality and mortality related to PE during index hospitalization and readmission periods. Economic outcomes included total hospitalization costs during index hospitalization and readmission periods and pharmacologic prophylaxis costs during the index hospitalization period. Pharmacologic prophylaxis costs included total costs 
for all prophylactic doses of enoxaparin and UFH and were determined from the hospitals' charge master data.

Major bleeding events and heparin-induced thrombocytopenia (HIT) during the index hospitalization and readmission periods were identified. A major bleeding event was defined by the presence of a primary or secondary admission or discharge ICD-9/10-CM diagnosis code for serious bleeding (ICD-9-CM: 430, 431, 432.x, 459.0, 578.x, 786.3x; ICD10-CM: I60.9-I62.9, R58, K92.0, K92.2, R04.x, D75.82). HIT was defined by the presence of a primary or secondary admission or discharge ICD-9-CM diagnosis code of 289.84 or ICD-10-CM diagnosis code of D75.82.

The study exposure was VTE prophylaxis with either enoxaparin or UFH. Patients were categorized based on receipt of at least one prophylactic dose (enoxaparin $\leq 40$ $\mathrm{mg}$ /day; UFH $\leq 15,000$ IU/day) during the index hospitalization as determined from the hospitals' charge master data. Patients who received VTE prophylactic doses of both enoxaparin and UFH were not included.

Several variables, including patient demographic, visit, clinical, and hospital characteristics, that might confound the relationship between enoxaparin prophylaxis and study outcomes were assessed during index hospitalization and included as covariates. Patient demographics included age, sex, race, and payer type. Visit characteristics included admission type and source, discharge disposition, ICU admissions, and hospital length of stay. Comorbidities identified by the presence of ICD-9/10 diagnoses codes during the index hospitalization included congestive heart failure (ICD9-CM: 428-428.9x; ICD-10-CM: I50.1, I50.20-I50.23, I50.33, I50.40-I50.43, I50.9), myocardial infarction (ICD9-CM: 410-410.9x, 412-412.9x; ICD-10-CM: I21.x, I22.x, I25.2, I23.x), chronic obstructive pulmonary disease (ICD9-CM: 490-496.9x, 500-505.9x, 506.4x; ICD-10-CM: J40, J41.x, J42, J43.9, J44.x, J45.2x, J45.90x, J45.99x, J47.1, J47.9, J60, J61, J62.8, J63.x, J66.x, J64, J67.x, J68.4), fracture of lower limb (ICD-9-CM: 820.x; ICD-10-CM: S82.x), inflammatory bowel disease (ICD-9-CM: 555-556.x; ICD10-CM: K50-K51.xxx), intubation (ICD-9-Procedure Classification System (PCS): I96.0x-96.5x, 96.6, 96.7x; ICD-10-PCS: 09Hхxхx, 099xхxx, 0BНxxxx, 0B9xxxx, 0D9xxxx, 0T7xxxx, 0UHxxxx, 0WHxxxx, 0YQxxxx), malignant hypertension (ICD-9-CM: 401.0, 402-405.xx; ICD-10-CM: I10, I1 1-I13.xx, I15.x), myocardial infarction (ICD-9-CM: 410-410.9x, 412-412.9x; ICD-10-CM: I21.x, I22.x, I25.2, I23.x), nephrotic syndrome (ICD-9-CM: 581. $\mathrm{xx}$; ICD-10-CM: N04.x), and obesity (ICD-9-CM: 278; ICD-10-CM: E66). In addition, 3M ${ }^{\mathrm{TM}}$ All Patient Refined ${ }^{\mathrm{TM}}$ Diagnosis-Related Group (APR ${ }^{\mathrm{TM}}$-DRG) Severity of Illness (APR-SOI) [19], and the Deyo modification of the Charlson Comorbidity Index (CCI) [20] were used to assess health status. The APR-SOI (minor, moderate, major, or extreme) was computed at the time of hospital discharge and accounts for age, procedures, and clinical severity of primary diagnosis and all secondary diagnoses assigned in the course of index hospitalization [19]. Hospital characteristics included teaching status, bed size, geographical region, and population served.

\subsection{Statistical Analysis}

Descriptive statistics were presented using mean \pm standard deviation (SD) for continuous variables and using frequencies and proportions for categorical variables. Bivariate analyses were conducted to assess differences in patient demographics and visit, clinical, and hospital characteristics between enoxaparin and UFH prophylaxis groups using Student's t-test or the Wilcoxon rank sum test for continuous variables and the $\chi^{2}$ test for categorical variables. Normality of the data was assessed by histogram and the Kolmogorov-Smirnov test to determine the appropriate tests to apply for statistical significance. Multivariable logistic regression models were conducted to estimate the odds of VTE events, in-hospital mortality, PE-related mortality, and major bleeding between enoxaparin and UFH prophylaxis groups during the index hospitalization and readmission periods. Economic outcomes, including total hospitalization costs and pharmacologic prophylaxis costs during the index hospitalization period, and total hospitalization costs during the readmission period, were reported using unadjusted mean \pm SD. Outliers were assessed and those $<1$ st percentile and $>99$ th percentile were excluded. Generalized linear models with gamma link function were constructed to estimate adjusted costs for each study group and presented using adjusted means and confidence intervals (CIs). Patients with missing cost data were excluded from the study. All regression models were assessed for fitness and convergence of algorithm. Regression diagnostics were conducted to assess multicollinearity between variables and did not warrant deletion of any variables. A $p<0.05$ was used for statistically significant inferences. Data management and analyses were performed using SAS v. 9.4 (SAS Institute Inc., Cary, NC, USA).

\section{Results}

A total of 376,858 medically ill inpatients met the study criteria, with $64 \%(242,474)$ receiving VTE prophylaxis with enoxaparin and $36 \%(134,384)$ receiving UFH during index hospitalization. Table 1 presents patient demographics and visit, clinical, and hospital characteristics for the two VTE prophylactic groups. Statistically significant differences in all variables were observed between the two study groups $(p<0.05)$. Compared with medically ill inpatients with UFH prophylaxis, those with enoxaparin prophylaxis were younger (mean age of $65 \pm 17$ vs. $67 \pm 16$ years), more 
likely to be female (55 vs. $50 \%$ ), predominantly White (74 vs. $68 \%$ ), and less likely to be Medicare beneficiaries (61 vs. $65 \%)(p<0.0001$ for each).

A significantly higher proportion of patients in the enoxaparin group compared with the UFH group were admitted emergently (75 vs. $72 \%$ ) from a non-healthcare facility (79 vs. $75 \%)$ and discharged to home (69 vs. $64 \%)(p<0.0001$ for each). The proportion of enoxaparin patients with extreme SOI (18 vs. $25 \%$ ) and a CCI score of $\geq 2$ (36 vs. $47 \%$ ) was significantly lower than that of UFH inpatients $(p<0.0001$ for each). Compared with the UFH group, a higher proportion of patients in enoxaparin group had a comorbid condition of chronic obstructive pulmonary disease ( 47 vs. $38 \%$ ) and malignant hypertension (10 vs. 9\%) ( $p<0.0001$ for each). Moreover, a greater proportion of enoxaparin patients compared with UFH patients were treated in non-teaching hospitals (62 vs. $44 \%$ ) of smaller bed capacity ( 36 vs. $31 \%$ ) located in the southern USA (54 vs. $34 \%$ ) that served rural populations (13 vs. $8 \%)(p<0.0001$ for each).

\subsection{Comparative Effectiveness}

Table 2 presents the clinical outcomes during the index hospitalization and readmission periods for the enoxaparin and UFH prophylaxis groups. During index hospitalization, VTE event, in-hospital mortality, and PE-related mortality rates were $0.47 \%, 3.58 \%$, and $0.03 \%$, respectively, for the enoxaparin group and $0.76 \%, 5.47 \%$, and $0.08 \%$, respectively, for the UFH group. Multivariable regression analyses revealed that enoxaparin prophylaxis was significantly associated with a lower odds of $15 \%$ for VTE events (odds ratio [OR] $0.85 ; p=0.001$ ), $9 \%$ for in-hospital mortality (OR 0.91 ; $p<0.0001$ ), and $33 \%$ for PE-related mortality (OR 0.67 ; $p=0.015)$ than UFH prophylaxis during the index hospitalization period. A total of 105,836 (44\%) in the enoxaparin group and 57,763 (43\%) in the UFH group were readmitted for any cause within 90 days after discharge from index hospitalization. Among readmitted patients, VTE event, in-hospital mortality, and PE-related mortality rates were $2.71 \%, 4.32 \%$, and $0.18 \%$ for the enoxaparin group and $3.15 \%, 4.90 \%$, and $0.15 \%$, respectively, for the UFH group. Compared with UFH prophylaxis, enoxaparin prophylaxis was significantly associated with a $10 \%$ lower odds of a VTE (OR 0.90; $p=0.0022$ ) and $8 \%$ higher odds of inpatient death (OR 1.08; $p=0.008)$ in readmitted inpatients. No statistically significant differences in PE-related mortality were observed.

\subsection{Major Bleeding and Heparin-Induced Thrombocytopenia}

During the index hospitalization, 5328 (2.2\%) patients in the enoxaparin group and $6281(4.7 \%)$ in the UFH group were coded with a major bleeding event $(p<0.0001)$. During the readmission period, 3161 (3\%) patients in the enoxaparin group and 2374 (4.1\%) in the UFH group were similarly coded $(p<0.0001)$. The enoxaparin group had a $41 \%$ lower risk of bleeding during the index encounter visit (OR 0.59; 95\% CI 0.57-0.62) and a 19\% lower risk in the 90-day follow-up period (OR 0.81 ; 95\% CI 0.77-0.86) than those treated with UFH. HIT was identified in $105(0.04 \%)$ patients in the enoxaparin group and $172(0.1 \%)$ patients in the UFH group $(p<0.0001)$ during the index hospitalization and $67(0.06 \%)$ patients in the enoxaparin group and 96 $(0.2 \%)$ patients in the UFH group $(p<0.0001)$ during the readmission period.

\subsection{Economic Outcomes}

Table 3 presents unadjusted and adjusted total hospitalization costs during index hospitalization and readmission periods and unadjusted and adjusted pharmacologic prophylaxis costs during the index hospitalization period. Compared with UFH prophylaxis, patients with enoxaparin prophylaxis had significantly lower adjusted mean total hospitalization costs during the index period (2014US\$15,665 [95\% CI 15,386-15,907] vs. 17,092 [95\% CI 16,887-17,320]; $p<0.0001)$ and during the readmission period (2014US\$5108 [95\% CI 4707-5544] vs. 5455 [95\% CI 5194-5921]; $p<0.0001$ ). Higher adjusted mean pharmacologic prophylaxis costs during index hospitalization were noted for enoxaparin compared with UFH (2014US\$140 [95\% CI 136-145] vs. 67 [95\% CI 65-69]; $p<0.0001$ ).

\section{Discussion}

A large hospital database was used to assess the real-world use of thromboprophylaxis with enoxaparin and UFH in hospitalized medically ill patients in the USA. Prophylaxis with enoxaparin versus UFH was associated with a statistically significant lower odds of VTE events, hospital deaths, deaths related to PE, and major bleeding during index hospitalization and of VTE events and major bleeding among patients readmitted within 90 days of index discharge. In addition, there was a lower adjusted mean hospitalization cost during index hospitalization and the readmission period for those in the enoxaparin versus UFH groups. Other studies have found inconsistent results, likely because of differences in study populations and designs.

Several randomized controlled trials comparing enoxaparin and UFH prophylaxis in non-surgical medical inpatients who were at risk of VTE found greater reductions in VTE events than observed in our study [14-17]. This may reflect the more homogenous populations of patients with 
Table 1 Patient demographic, clinical, and hospital characteristics of medically ill patients on enoxaparin versus unfractionated heparin prophylaxis

\begin{tabular}{|c|c|c|c|}
\hline Characteristics & $\begin{array}{l}\text { Enoxaparin prophylaxis } \\
(N=242,474)\end{array}$ & $\begin{array}{l}\text { Unfractionated heparin } \\
\text { prophylaxis } \\
(N=134,384)\end{array}$ & $p$-Value \\
\hline \multicolumn{4}{|l|}{ Patient demographic characteristics } \\
\hline Age (years) & $65 \pm 17$ & $67 \pm 16$ & $<0.0001$ \\
\hline Female sex & $132,994(55)$ & $67,050(50)$ & $<0.0001$ \\
\hline \multicolumn{4}{|l|}{ Race } \\
\hline White & $179,776(74)$ & $91,357(68)$ & \multirow[t]{4}{*}{$<0.0001$} \\
\hline Black & $29,059(12)$ & $19,114(14)$ & \\
\hline Other & $32,642(14)$ & $23,551(18)$ & \\
\hline Unknown & $997(0.4)$ & $362(0.3)$ & \\
\hline \multicolumn{4}{|l|}{ Payer type } \\
\hline Private & $47,395(20)$ & $25,653(19)$ & \multirow[t]{5}{*}{$<0.0001$} \\
\hline Medicaid & $28,157(12)$ & $14,640(11)$ & \\
\hline Medicare & $147,754(61)$ & $86,792(65)$ & \\
\hline Uninsured & $15,631(7)$ & $5927(4)$ & \\
\hline Unknown & $3537(2)$ & $1372(1)$ & \\
\hline \multicolumn{4}{|l|}{ Visit characteristics } \\
\hline \multicolumn{4}{|l|}{ Admission source } \\
\hline Non-healthcare facility of origin & $192,579(79)$ & $100,534(75)$ & \multirow[t]{5}{*}{$<0.0001$} \\
\hline Transfer from acute care facility & $20,245(8)$ & $17,243(13)$ & \\
\hline Transfer from long-term care facility & $5991(3)$ & $4216(3)$ & \\
\hline Clinic/emergency room & $15,125(6)$ & $7350(6)$ & \\
\hline Other/unknown & $8534(4)$ & $5041(4)$ & \\
\hline \multicolumn{4}{|l|}{ Admission type } \\
\hline Emergency & $181,114(75)$ & $96,399(72)$ & \multirow[t]{4}{*}{$<0.0001$} \\
\hline Urgent & $37,858(16)$ & $21,133(16)$ & \\
\hline Elective & $21,567(9)$ & $15,611(12)$ & \\
\hline Trauma & $786(0.3)$ & $629(0.5)$ & \\
\hline \multicolumn{4}{|l|}{ Discharge status } \\
\hline Expired & $8673(4)$ & $7350(6)$ & \multirow[t]{5}{*}{$<0.0001$} \\
\hline Home & $166,515(69)$ & $86,070(64)$ & \\
\hline Transferred to another acute care & $3849(2)$ & $2434(2)$ & \\
\hline Transferred to nursing or rehabilitation facility & $59,602(25)$ & $36,178(27)$ & \\
\hline Other/unknown & $3835(2)$ & $2352(2)$ & \\
\hline ICU admission stay & $62,993(26)$ & $45,683(34)$ & $<0.0001$ \\
\hline Hospital length of stay (days) & $8.7 \pm 4.6$ & $9.1 \pm 5.9$ & $<0.0001$ \\
\hline \multicolumn{4}{|l|}{ Clinical characteristics } \\
\hline Severity of illness & & & $<0.0001$ \\
\hline Minor & $14,968(6)$ & $7439(6)$ & \\
\hline Moderate & $73,587(30)$ & $32,914(25)$ & \\
\hline Major & $109,374(45)$ & $60,781(45)$ & \\
\hline Extreme & $44,545(18)$ & $33,250(25)$ & \\
\hline Charlson Comorbidity Index Score ${ }^{\mathrm{a}}$ & & & $<0.0001$ \\
\hline 0 & $34,326(14)$ & $15,447(12)$ & \\
\hline $1-2$ & $121,194(50)$ & $55,862(42)$ & \\
\hline$\geq 2$ & $86,954(36)$ & $63,075(47)$ & \\
\hline Congestive heart failure & $58,488(24)$ & $42,726(32)$ & $<0.0001$ \\
\hline COPD & $114,174(47)$ & $51,011(38)$ & $<0.0001$ \\
\hline Fracture of lower limb & $706(0.3)$ & $287(0.2)$ & $<0.0001$ \\
\hline
\end{tabular}


Table 1 (continued)

\begin{tabular}{|c|c|c|c|}
\hline Characteristics & $\begin{array}{l}\text { Enoxaparin prophylaxis } \\
(N=242,474)\end{array}$ & $\begin{array}{l}\text { Unfractionated heparin } \\
\text { prophylaxis } \\
(N=134,384)\end{array}$ & $p$-Value \\
\hline Inflammatory bowel disease & $1776(1)$ & $882(1)$ & 0.0075 \\
\hline Intubation & $21,983(9)$ & $16,029(12)$ & $<0.0001$ \\
\hline Malignant hypertension & $23,738(10)$ & $11,597(9)$ & $<0.0001$ \\
\hline Myocardial infarction & $19,413(9)$ & $15,044(11)$ & $<0.0001$ \\
\hline Nephrotic syndrome & $202(0.1)$ & $242(0.2)$ & $<0.0001$ \\
\hline Obesity & $44,420(18)$ & $22,941(17)$ & $<0.0001$ \\
\hline \multicolumn{4}{|l|}{ Hospital characteristics } \\
\hline Geographic region & & & $<0.0001$ \\
\hline Northeast & $37,392(15)$ & $46,864(35)$ & \\
\hline Midwest & $42,200(17)$ & $25,048(19)$ & \\
\hline South & $130,490(54)$ & $45,616(34)$ & \\
\hline West & $32,392(13)$ & $16,856(13)$ & \\
\hline Bed size & & & $<0.0001$ \\
\hline $1-299$ & $87,453(36)$ & $40,926(31)$ & \\
\hline $300-499$ & $78,027(32)$ & $45,961(34)$ & \\
\hline$\geq 500$ & $76,994(32)$ & $47,497(35)$ & \\
\hline Population served & & & $<0.0001$ \\
\hline Rural & $31,489(13)$ & $10,314(8)$ & \\
\hline Urban & $210,985(87)$ & $124,070(92)$ & \\
\hline Teaching & & & $<0.0001$ \\
\hline Non-teaching & $150,371(62)$ & $58,685(44)$ & \\
\hline Teaching & $92,103(38)$ & $75,699(56)$ & \\
\hline
\end{tabular}

Data are presented as mean \pm standard deviation or $\mathrm{N}(\%)$ unless otherwise indicated

$C O P D$ chronic obstructive pulmonary disease, $I C U$ intensive care unit

${ }^{a}$ Myocardial infarction, congestive heart failure, peripheral vascular disease, history of cerebrovascular accident and transient ischemic attacks, dementia, COPD, connective tissue disease, mild or moderate to severe liver disease, diabetes mellitus uncomplicated or with end-organ damage, hemiplegia, mild or moderate to severe renal disease, malignancy, and HIV positive status

Table 2 Clinical outcomes during index hospitalization and 90-day readmission periods for medically ill patients on enoxaparin and unfractionated heparin prophylaxis

Unfractionated heparin prophylaxis
Enoxaparin prophylaxis (vs. unfractionated heparin prophylaxis)

$\mathrm{OR}^{\mathrm{a}}(95 \% \mathrm{CI}) \quad p$-Value

Index hospitalization period events

$\begin{array}{lll}\text { VTE event } & 1144(0.47) & 1017(0.76) \\ \text { In-hospital mortality } & 8673(3.58) & 7350(5.47) \\ \text { PE-related mortality } & 79(0.03) & 109(0.08) \\ \text { 90-day readmission period events } & & 1817(3.15) \\ \text { VTE event } & 2867(2.71) & 2831(4.90) \\ \text { In-hospital mortality } & 4572(4.32) & 103(0.15) \\ \text { PE-related mortality } & 154(0.18) & \end{array}$

Data are presented as n (\%) unless otherwise indicated

$C I$ confidence interval, $I C U$ intensive care unit, $O R$ odds ratio, $P E$ pulmonary embolism, VTE venous thromboembolism

${ }^{a}$ OR adjusted for patient characteristics (age, sex, race, payer); visit characteristics (admission source and type, and ICU admission); clinical characteristics (severity of illness, Charlson Comorbidity Index score, obesity, myocardial infarction, congestive heart failure, chronic obstructive pulmonary disease, inflammatory bowel disease, intubation, fracture of lower limb, nephrotic syndrome, and malignant hypertension); and hospital characteristics (teaching status, bed size, geographic region, and rurality) 
Table 3 Economic outcomes during index hospitalization and 90-day readmission periods for medically ill patients on enoxaparin and unfractionated heparin prophylaxis

\begin{tabular}{|c|c|c|c|c|c|}
\hline & \multicolumn{3}{|c|}{ Unadjusted mean costs } & \multicolumn{2}{|l|}{ Adjusted mean estimates ${ }^{\mathrm{a}}$} \\
\hline & $\begin{array}{l}\text { Enoxaparin prophy- } \\
\text { laxis }\end{array}$ & $\begin{array}{l}\text { Unfractionated } \\
\text { heparin prophy- } \\
\text { laxis }\end{array}$ & $p$-Value & $\begin{array}{l}\text { Enoxaparin prophy- } \\
\text { laxis }\end{array}$ & $\begin{array}{l}\text { Unfractionated heparin } p \text {-Value } \\
\text { prophylaxis }\end{array}$ \\
\hline \multicolumn{6}{|c|}{ Index hospitalization period costs } \\
\hline Total hospitalization & $15,814 \pm 12,953$ & $20,282 \pm 19,754$ & $p<0.0001$ & $15,665(15,386-15,907)$ & $17,092(16,887-17,320) p<0.0001$ \\
\hline $\begin{array}{l}\text { Pharmacologic } \\
\text { prophylaxis }\end{array}$ & $178 \pm 190$ & $86 \pm 121$ & $p<0.0001$ & $140(136-145)$ & $67(65-69) \quad p<0.0001$ \\
\hline \multicolumn{6}{|c|}{ 90-day readmission period costs } \\
\hline Total hospitalization & $9774 \pm 20,449$ & $12,270 \pm 30,392$ & $p<0.0001$ & $5108(4707-5544)$ & 5455 (5194-5921) $p<0.0001$ \\
\hline
\end{tabular}

Data are presented in 2014US\$ as mean \pm standard deviation or mean (95\% confidence interval) unless otherwise indicated

$C I$ confidence interval, $I C U$ intensive care unit

${ }^{a}$ Adjusted variables include patient characteristics (age, sex, race, payer); visit characteristics (admission source and type, and ICU admission); clinical characteristics (severity of illness, Charlson Comorbidity Index score, obesity, myocardial infarction, congestive heart failure, chronic obstructive pulmonary disease, inflammatory bowel disease, intubation, fracture of lower limb, nephrotic syndrome, and malignant hypertension); and hospital characteristics (teaching status, bed size, geographic region, and rurality)

heart failure, severe respiratory disease, and acute ischemic stroke in the respective trials. In contrast, a recent metaanalysis [21] did not find a significant reduction in VTE events between enoxaparin and UFH prophylaxis in medically ill inpatients. This meta-analysis evaluated the efficacy of all VTE prophylactic drugs in medically ill patients but included only one study [16] for direct comparison and three studies [22-24] for indirect comparisons between enoxaparin and UFH prophylaxis. Randomized clinical trials assessing VTE prophylaxis have reported inconsistent findings for reduced bleeding with enoxaparin compared with heparin. Similar to our study, a multicenter, controlled, randomized, open study of medical patients with heart failure or severe respiratory disease demonstrated less bleeding with enoxaparin [14]. A randomized double-blind study of ischemic stroke patients with lower limb paralysis resulting in bed rest also showed that enoxaparin was associated with a reduced risk of bleeding [17]. In contrast, the PREVAIL open-label study of patients with acute ischemic stroke unable to walk unassisted showed no difference in the occurrence of any bleeding between enoxaparin and UFH treatments [15].

Few studies using large real-world hospital or administrative databases have been conducted to compare effectiveness between enoxaparin and UFH thromboprophylaxis in heterogeneous medically ill inpatients [25, 26]. A $49 \%$ and $74 \%$ lower risk of VTE events was reported in two studies, which is substantially higher than the $15 \%$ reduced risk in the current study $[25,26]$. Differences in the age and SOI of the study cohorts as compared with the current study might explain the greater reduction in odds of VTE events. Moreover, previous studies that investigated the association of enoxaparin prophylaxis with hospital death during index hospitalization did not find significant results [21, 25], unlike the current study where a $9 \%$ reduced odds of hospital deaths was observed. A smaller study cohort in McGarry et al. [25], and use of direct and indirect comparisons in the meta-analysis by $\mathrm{Al}$ Yami et al. [21], may partially explain the differences in findings.

In this study, enoxaparin prophylaxis was also associated with lower total hospitalization costs. Despite higher medication costs, the mean adjusted total index hospital cost for the enoxaparin prophylaxis group was 2014US\$1427 lower than that for the UFH group. Given that it costs around 2014US\$7712-16,644 to manage one VTE event in the USA [27], thromboprophylaxis with enoxaparin might reduce the economic burden in medically ill patients at risk of VTE. This economic benefit is consistent with that observed in previous studies comparing enoxaparin and UFH [26, 28-32]. Nonetheless, the clinical significance of the association between enoxaparin and costs should be interpreted with care as the UFH cohort had longer hospitalization stays. Longer lengths of stay, major and extreme SOI, and higher CCI score could reflect a sicker status than that of the enoxaparin patients. While VTE prophylaxis guidelines generally recommend LMWH as the drug of choice for medically ill hospitalized patients, UFH is indicated in patients with renal failure (creatinine clearance $<30 \mathrm{ml} / \mathrm{min}$ ). UFH has a short half-life, is predominately metabolized by the liver, and generally does not require dose adjustment with renal failure as does LMWH, particularly enoxaparin [33, 34].

The potentially beneficial effects of enoxaparin prophylaxis were also seen during the 90 days following discharge from index hospitalization. Readmitted patients who received enoxaparin thromboprophylaxis during index hospitalization had significantly fewer VTE events and lower total hospitalization costs in the readmission period than 
those who received UFH during the index hospitalization. Real-world evidence studies comparing clinical and economic readmission outcomes are limited [32, 35]. Kociol et al. [35] found no statistically significant differences in 30-day post discharge outcomes in patients with heart failure, whereas a decision-analysis study by Deitelzweig et al. [32] reported findings favorable for enoxaparin prophylaxis for 2-year readmission outcomes and costs. The current study included only patients readmitted to the same index hospital within 90 days after discharge. In addition, no variables in the readmission period were captured to adjust for in the models. In addition, differences with earlier studies in the nature of study patients, readmission time period, and primary pharmacologic thromboprophylactic comparison groups $[32,35]$ make comparisons with the current study challenging.

This study has several limitations, many related to the inherent characteristics of a hospital administrative database. First the PHD is a hospital administrative database that relies on ICD codes and does not contain the many clinical details and laboratory values available from electronic healthcare records. Clinical details associated with VTE risk such as smoking status, prior VTE history, underlying malignancy, recent trauma/surgery, and immobility were not available, and review of medical charts was outside the scope of this study. Second, laboratory creatine clearance values were also unavailable, and it is difficult to determine whether use of UFH could be attributed to renal failure. Nonetheless, renal disease was determined by ICD codes and included in the CCI score. Thus, the possibility of unmeasured confounding or residual confounding cannot be ruled out. Lastly, inaccurate or incomplete ICD-9/10-CM diagnosis coding by hospitals could have resulted in misidentification of study-eligible patients, covariates, or VTE and PE events. It was not possible to distinguish between symptomatic and asymptomatic VTE events since VTE diagnostic testing was not captured in the PHD. As in all retrospective observational studies, patients were not randomly assigned to drug treatment, which could result in selection bias. Although efforts were taken to capture VTE events after the receipt of prophylactic drugs and to exclude subjects with VTE diagnosis within the first 2 days of index hospitalization admission, the study was not designed to determine causal relationships. Events in the follow-up period might have been underestimated because subsequent encounters may have occurred outside the index hospital. While many of the described limitations are associated with data source and study design, use of realworld data from a large geographically diverse hospital database reflects the current state of clinical and prophylactic prescribing practices with enoxaparin and UFH to prevent VTE in hospitalized medically ill patients. Moreover, studies using real-world evidence to assess clinical and economic outcomes are scarce. These findings add to the clinical trial data that are designed under very controlled conditions.

\section{Conclusions}

This study provides a contemporary, real-world, US hospital-based perspective of enoxaparin and UFH thromboprophylaxis in diverse, medically ill, hospitalized patients. Enoxaparin prophylaxis was associated with a statistically significant lower odds of VTE events, in-hospital mortality, PE-related mortality, and major bleeding than was UFH. Our results highlight the need to identify patients at high risk for VTE and opportunities to optimize the selection of the appropriate thromboprophylaxis.

Acknowledgements Ms. Carol Cohen, senior medical writer employed by Premier Applied Sciences, Premier Inc., provided publication support. Dr. Ning Rosenthal, from Premier Applied Sciences, Premier Inc., provided statistical input and analyses. Dr. Jenny Shan at Sanofi, Inc. provided clinical input during revision of the manuscript. Wilson Ngai at Sanofi, Inc provided review and valuable feedback on the manuscript draft.

\section{Declarations}

Funding This study was funded by Sanofi US Services, Inc.

Conflict of Interest S.P. Veeranki was an employee of Premier Applied Sciences, Premier Inc. at the time of the study. M. Sinha is an employee of Premier Applied Sciences, Premier, Inc. Premier Applied Sciences received funding from Sanofi US Services, Inc. to conduct this study. B. Shah is employed by and holds equity from Livongo Health and also serves as a consultant for Premier Inc., Janssen, and Medtronic. Z. Xiao was an employee of Sanofi at the time of the study and is a stockholder. A. Levorsen is an employee of and stockholder in Sanofi.

Ethics Approval The PHD is considered exempt from Institutional Review Board oversight as dictated by Title 45 Code of Federal Regulations, Part 46 of the United States, specifically 45 CFR 46.101(b) (4). In accordance with the HIPAA Privacy Rule, disclosed data from the PHD are considered de-identified per 45 CFR 164.506(d)(2)(ii)(B) through the "Expert Determination" method.

Consent to participate Not applicable.

Availability of data and material Data from the PHD are proprietary and cannot be shared externally.

Code availability Not applicable.

Authors' contributions S.P. Veeranki, Z. Xiao, and A. Levorsen were responsible for design of the study. S.P. Veeranki and M. Sinha had full access to all the data in the study and take responsibility for the integrity of the data and accuracy of the data analysis. S.P. Veeranki interpreted the study findings with assistance from Z. Xiao and B. Shah and drafted the first draft of the manuscript. All authors were responsible for critical revision of the manuscript, for important intellectual content, and provided approval of the final draft. 
Open Access This article is licensed under a Creative Commons Attribution-NonCommercial 4.0 International License, which permits any non-commercial use, sharing, adaptation, distribution and reproduction in any medium or format, as long as you give appropriate credit to the original author(s) and the source, provide a link to the Creative Commons licence, and indicate if changes were made. The images or other third party material in this article are included in the article's Creative Commons licence, unless indicated otherwise in a credit line to the material. If material is not included in the article's Creative Commons licence and your intended use is not permitted by statutory regulation or exceeds the permitted use, you will need to obtain permission directly from the copyright holder. To view a copy of this licence, visit http://creativecommons.org/licenses/by-nc/4.0/.

\section{References}

1. Grosse SD, Nelson RE, Nyarko KA, Richardson LC, Raskob GE. The economic burden of incident venous thromboembolism in the United States: a review of estimated attributable healthcare costs. Thromb Res. 2016;137:3-10. https://doi.org/10.1016/j. thromres.2015.11.033.

2. The surgeon general's call to action to prevent deep vein thrombosis and pulmonary embolism. Rockville (MD). 2008.

3. Al Yami MS, Silva MA, Donovan JL, Kanaan AO. Venous thromboembolism prophylaxis in medically ill patients: a mixed treatment comparison meta-analysis. J Thromb Thrombolysis. 2018;45:36-47. https://doi.org/10.1007/s11239-017-1562-5.

4. Lenchus JD, Jaffer AK. Prevention of venous thromboembolism in hospitalized medical patients. Mt Sinai J Med. 2008;75:449-53. https://doi.org/10.1002/msj.20074.

5. Edelsberg J, Hagiwara M, Taneja C, Oster G. Risk of venous thromboembolism among hospitalized medically ill patients. Am J Health Syst Pharm. 2006;63:S16-22. https://doi.org/10.2146/ ajhp060389.

6. Cave B, Hough A, Dobesh PP. Extended venous thromboembolism prophylaxis in medically ill patients. Pharmacotherapy. 2018;38:597-609. https://doi.org/10.1002/phar.2102.

7. Prevention CfDCa. National Center for Health Statistics. Emergency Department Visits. 2018.

8. Schunemann HJ, Cushman M, Burnett AE, Kahn SR, BeyerWestendorf J, Spencer FA, Rezende SM, Zakai NA, Bauer KA, Dentali F, Lansing J, Balduzzi S, Darzi A, Morgano GP, Neumann I, Nieuwlaat R, Yepes-Nunez JJ, Zhang Y, Wiercioch W. American Society of Hematology 2018 guidelines for management of venous thromboembolism: prophylaxis for hospitalized and nonhospitalized medical patients. Blood Adv. 2018;2:3198-225. https ://doi.org/10.1182/bloodadvances.2018022954.

9. Kearon C, Akl EA, Ornelas J, Blaivas A, Jimenez D, Bounameaux H, Huisman M, King CS, Morris TA, Sood N, Stevens SM, Vintch JRE, Wells P, Woller SC, Moores L. Antithrombotic therapy for VTE disease: CHEST guideline and expert panel report. Chest. 2016;149:315-52. https://doi.org/10.1016/j.chest.2015.11.026.

10. Dentali F, Douketis JD, Gianni M, Lim W, Crowther MA. Metaanalysis: anticoagulant prophylaxis to prevent symptomatic venous thromboembolism in hospitalized medical patients. Ann Intern Med. 2007;146:278-88.

11. Lloyd NS, Douketis JD, Moinuddin I, Lim W, Crowther MA. Anticoagulant prophylaxis to prevent asymptomatic deep vein thrombosis in hospitalized medical patients: a systematic review and meta-analysis. J Thromb Haemost. 2008;6:405-14. https:// doi.org/10.1111/j.1538-7836.2007.02847.x.

12. Sjalander A, Jansson JH, Bergqvist D, Eriksson H, Carlberg B, Svensson P. Efficacy and safety of anticoagulant prophylaxis to prevent venous thromboembolism in acutely ill medical inpatients: a meta-analysis. J Intern Med. 2008;263:52-60. https://doi.org/1 0.1111/j.1365-2796.2007.01878.x.

13. Laporte S, Liotier J, Bertoletti L, Kleber FX, Pineo GF, Chapelle C, Moulin N, Mismetti P. Individual patient data meta-analysis of enoxaparin vs. unfractionated heparin for venous thromboembolism prevention in medical patients. J Thromb Haemost. 2011;9:464-72. https://doi.org/10.1111/j.1538-7836.2011.04182 .x.

14. Kleber FX, Witt C, Vogel G, Koppenhagen K, Schomaker U, Flosbach CW, Group T-PS. Randomized comparison of enoxaparin with unfractionated heparin for the prevention of venous thromboembolism in medical patients with heart failure or severe respiratory disease. Am Heart J. 2003;145:614-21. https://doi. org/10.1067/mhj.2003.189.

15. Sherman DG, Albers GW, Bladin C, Fieschi C, Gabbai AA, Kase CS, O'Riordan W, Pineo GF, Investigators P. The efficacy and safety of enoxaparin versus unfractionated heparin for the prevention of venous thromboembolism after acute ischaemic stroke (PREVAIL Study): an open-label randomised comparison. Lancet. 2007;369:1347-55. https://doi.org/10.1016/S0140 -6736(07)60633-3.

16. Lechler E, Schramm W, Flosbach CW. The venous thrombotic risk in non-surgical patients: epidemiological data and efficacy/ safety profile of a low-molecular-weight heparin (enoxaparin). The Prime Study Group. Haemostasis. 1996;26(Suppl 2):49-56. https://doi.org/10.1159/000217272.

17. Hillbom M, Erila T, Sotaniemi K, Tatlisumak T, Sarna S, Kaste M. Enoxaparin vs heparin for prevention of deep-vein thrombosis in acute ischaemic stroke: a randomized, double-blind study. Acta Neurol Scand. 2002;106:84-92.

18. Premier Healthcare Database White Paper. 2020. http://learn .premierinc.com/white-papers/premier-healthcare-database-white paper.

19. Averill RGN, Hughes J, Muldoon J, Gay J, McCullough E, Bonazelli J, Mullin R. What are apr-drgs? An introduction to severity of illness and risk of mortality adjustment methodology. 2020. https://www.hcup-us.ahrq.gov/db/nation/nis/APR-DRGsV20Met hodologyOverviewandBibliography.pdf. Accessed 28 April 2020

20. Deyo RA, Cherkin DC, Ciol MA. Adapting a clinical comorbidity index for use with ICD-9-CM administrative databases. J Clin Epidemiol. 1992;45:613-9.

21. Al Yami MS, McBride A, Katragadda C, Martin JR, Badreldin HA, Mohammed AH, Elmubark AM, Alzahrani MY, Alsheri AM, Abraham I. Correction to: Direct oral anticoagulants for the treatment of venous thromboembolism in patients with active malignancy: a systematic review and meta-analysis. J Thromb Thrombolysis. 2018. https://doi.org/10.1007/s11239-018-1777-0.

22. Samama MM, Cohen AT, Darmon JY, Desjardins L, Eldor A, Janbon C, Leizorovicz A, Nguyen H, Olsson CG, Turpie AG, Weisslinger $\mathrm{N}$. A comparison of enoxaparin with placebo for the prevention of venous thromboembolism in acutely ill medical patients. Prophylaxis in Medical Patients with Enoxaparin Study Group. N Engl J Med. 1999;341:793-800. https://doi.org/10.1056/ NEJM199909093411103.

23. Fraisse F, Holzapfel L, Couland JM, Simonneau G, Bedock B, Feissel M, Herbecq P, Pordes R, Poussel JF, Roux L. Nadroparin in the prevention of deep vein thrombosis in acute decompensated COPD. The association of non-University Affiliated Intensive Care Specialist Physicians of France. Am J Respir Crit Care Med. 2000;161:1109-14. https://doi.org/10.1164/ajrccm.161.4.9807025.

24. Harenberg J, Roebruck P, Heene DL. Subcutaneous low-molecular-weight heparin versus standard heparin and the prevention of thromboembolism in medical inpatients. The Heparin Study in Internal Medicine Group. Haemostasis. 1996;26:127-39. https:// doi.org/10.1159/000217198. 
25. McGarry LJ, Stokes ME, Thompson D. Outcomes of thromboprophylaxis with enoxaparin vs. unfractionated heparin in medical inpatients. Thromb J. 2006;4:17. https://doi. org/10.1186/1477-9560-4-17.

26. Amin AN, Lin J, Lenhart G, Schulman KL. Clinical and economic outcomes in patients at risk of venous thromboembolism receiving appropriate enoxaparin or unfractionated heparin prophylaxis. Thromb Haemost. 2009;102:321-6. https://doi.org/10.1160/ TH09-03-0147.

27. Dobesh PP. Economic burden of venous thromboembolism in hospitalized patients. Pharmacotherapy. 2009;29:943-53. https ://doi.org/10.1592/phco.29.8.943.

28. Pineo G, Lin J, Stern L, Subrahmanian T, Annemans L. Economic impact of enoxaparin versus unfractionated heparin for venous thromboembolism prophylaxis in patients with acute ischemic stroke: a hospital perspective of the PREVAIL trial. J Hosp Med. 2012;7:176-82. https://doi.org/10.1002/jhm.968.

29. McGarry LJ, Thompson D, Weinstein MC, Goldhaber SZ. Cost effectiveness of thromboprophylaxis with a low-molecular-weight heparin versus unfractionated heparin in acutely ill medical inpatients. Am J Manag Care. 2004;10:632-42.

30. Burleigh E, Wang C, Foster D, Heller S, Dunn D, Safavi K, Griffin B, Smith J. Thromboprophylaxis in medically ill patients at risk for venous thromboembolism. Am J Health Syst Pharm. 2006;63:S23-9. https://doi.org/10.2146/ajhp060390.

31. Offord R, Lloyd AC, Anderson P, Bearne A. Economic evaluation of enoxaparin for the prevention of venous thromboembolism in acutely ill medical patients. Pharm World Sci. 2004;26:214-20.

32. Deitelzweig SB, Becker R, Lin J, Benner J. Comparison of the two-year outcomes and costs of prophylaxis in medical patients at risk of venous thromboembolism. Thromb Haemost. 2008;100:810-20.

33. 33Pai M, Douketis JD. Prevention of venous thromboembolic disease in acutely ill hospitalized medical adult. UpToDate ${ }^{\circledR} .2020$. https://www.uptodate.com/contents/prevention-of-venous-throm boembolic-disease-in-acutely-ill-hospitalized-medical-adults. Accessed 23 Oct 2020.

34. Lowinger JS, Maxwll DJ. Heparins for venous thromboembolism prophylaxis—safety issues. Austr Prescriber. 2009;32:108-12.

35. Kociol RD, Hammill BG, Hernandez AF, Klaskala W, Mills RM, Curtis LH, Fonarow GC. Pharmacologic prophylaxis for venous thromboembolism and 30-day outcomes among older patients hospitalized with heart failure: an analysis from the ADHERE national registry linked to Medicare claims. Clin Cardiol. 2011;34:682-8. https://doi.org/10.1002/clc.20986.

\title{
Affiliations
}

\author{
S. Phani Veeranki ${ }^{1,2,7} \cdot$ Zhimin Xiao $^{3,8} \cdot$ Andrée Levorsen $^{4}\left({ }^{10} \cdot\right.$ Meenal Sinha $^{1} \cdot$ Bimal R. Shah $^{5,6}$ \\ S. Phani Veeranki \\ Phani.Veeranki@precisionvh.com \\ Zhimin Xiao \\ zxiao@post.harvard.edu \\ Premier Applied Sciences, Premier Inc., Charlotte, NC, USA \\ 2 Department of Preventive Medicine and Community Health, \\ University of Texas Medical Branch, Galveston, TX, USA \\ 3 Sanofi, Cambridge, MA, USA \\ 4 Global Health Economics and Value Assessment, Sanofi, \\ Professor Kohtsvei 5-17, Lysaker, 1366 Oslo, Norway \\ 5 Livongo Health, Mountain View, CA, USA \\ 6 Department of Medicine, Duke University, Durham, NC, \\ USA \\ 7 Precision HEOR, 11100 Santa Monica Blvd., Suite 500, \\ Los Angeles, CA, USA \\ 8650 E Kendall St, Cambridge, MA 02138, USA
}

